Will, Hart aber Fair, Markus Lanz, Beckmann, Kerner, usw.

87 Kepplinger, in Fischer, Lexikon, S. 700.

88 Kepplinger, in Faulbaum, Wolf, S. 111, 112.

89 Die folgenden Absätze beziehen sich auf Kepplinger, in Faulbaum/Wolf, S. 112, 119, 124.

90 Die folgenden Absätze beziehen sich auf Kepplinger, in Fischer, Lexikon, S. $659 \mathrm{ff}$

91 Muzafer Sherif, autokinetisches Phänomen 1935.

92 Kepplinger, Mechanismen, S. 23 ff.

93 Christian Schütze, früherer Redakteur der Süddeutschen Zeitung, Schütze, S. 34.

94 http://www.bild.de/BILD/regional/duesseldorf/aktuell/2011/02/21/mordfall-mirco/verteidiger-gerd-meister-erhaelt-drohbriefe.html, Stand Mai 2011, Auszug aus dem Bericht: „Hoffentlich besucht Euch Mirco in Euren Träumen, ihr Hundesöhne“, schreibt Gregor W. und weiter: „Wie viel Honorar bekommt man für einen Kindermörder? Gibt es Zuschläge oder 'ne Golftasche extra, Ihr Bastarde? ..."

95 „Deutschland schafft sich ab“ von Thilo Sarrazin.

96 Kepplinger in „Zur Sache Sarrazin“, S. 21.

97 Beckmann am 30.08.2010 und Hart aber Fair Sendung vom 01.09.2010.

98 Asch, S. 31-35.
99 Noelle-Neumann, S. 418 ff., S. 420; Schenk, S. $57 \mathrm{ff}$.

100 Welt online 16.02.2008 „Politiker wollen härtere Strafen für Steuersünder“, „Die Große Koalition will die Strafen für Steuersünder verschärfen. Das machten Union und SPD als Konsequenz aus dem Skandal um den scheidenden Post-Chef Klaus Zumwinkel deutlich. $\mathrm{Zu}$ oft würden Verfahren gegen Geldbuße eingestellt statt Anklage zu erheben, prangerte das SPD-Präsidium an. ...", Stern-Interview mit Kurt Beck vom 20.02.2008.

101 Interview mit Beulke, Prof. für Strafrecht, Strafprozessrecht und Kriminologie an der Universität Passau, Fokus online vom 18.02.2008, „Steuerskandal, Rechtsstaat auf dünnem Eis“.

102 So auch Neuling, S. 337.

103 Aktuell zu sehen an den verschiedenen jugendlichen „U-Bahnschlägern“, bei denen empörend reagiert wurde, dass ein Haftgrund für die U-Haft nicht gegeben war und die Anlass gaben, erneut den sog. "Warnschussarrest“ für Jugendliche zu fordern, z.B. Bild-Zeitung http://www.bild.de/regional/berlin/berlin-aktuell/politiker-fordern-warnschluss-arrestfuer-schlaeger-17619552.bild.html.

104 Vera Brübne soll gemeinsam mit Ferbach 1960 einen Doppelmord aus Habgier begangen haben. Trotz erheblicher Zweifel und schwacher Beweislage wurden sie 1962 verurteilt und Brühne 1979 begnadigt. Bereits vor Beginn des Prozesses wurde Brühne im Stern, in der Abendzeitung und in anderen Medien als Schuldige dargestellt. Über das Gerichtsverfahren wurde in der Boulevardpresse wochenlang berichtet, die attraktive Brühne als „geldgieriges Luder“ dargestellt und über - zur damaligen Zeit - skandalöse erotische Ausschweifungen spekuliert. http://www.spiegel.de/spiegel/print/d-41972665.html, Stand Mai 2011 „Abziehbilder von spätem Sex und Dolce vita bestimmten die Szenerie vor dem Schwurgericht, beflügelten Vorurteile, standen der Wahrheitsfindung im Wege -- und lockten das Publikum an, zu Tausenden."

105 Kepplinger, in von Schlieffen, S. 49, 50. Fn. bezieht sich auf die beiden folgenden Absätze.

106 Am 23.11.2000 titelte die Bild Zeitung auf der Titelseite „Neonazis ertränken Kind. Am helllichten Tag im Schwimmbad. Keiner half. Und eine ganze Stadt hat es totgeschwiegen“. Wahr war, dass der sechs jährige Joseph, Sohn eines deutsch-irakischen Apotheker Ehepaares, 1997 im Schwimmbad ertrunken war.

107 Die Zeit online, vom 25.11.2010 „Der Niederschlag von Sebnitz“ von Martin Machowecz.

\title{
(Fehl-)Informationen über Tatverdächtige und Opfer im Ermittlungsverfahren oder wie der „Fall Brunner“ medial instrumentalisiert wurde'
}

Heribert Ostendorf

\section{Die Fehlinformationen}

Im „Fall Brunner“ haben die Polizei und die Staatsanwaltschaft den Medien gegenüber zwei wichtige Informationen zunächst zurückgehalten, obwohl ansonsten detailliert über den Fall informiert wurde. Einmal wurde erst in der Hauptverhandlung bekannt, dass das Opfer unter Herzbeschwerden litt und diese möglicherweise zu dem Tod beigetragen haben, zum anderen und noch gewichtiger, dass der Getötete der in der S-Bahn von den Angeklagten angezettelten Auseinandersetzung und Bedrohung und nach dem Verlassen der S-Bahn die Angeklagten auf dem Bahnhofsgelände nochmals zur Rede gestellt und den ersten Schlag getätigt hatte. Dies waren für die soziale Bewertung des Geschehens bedeutsame Tatumstände, unabhängig davon, wie sie das Gericht später im Urteil gewürdigt hat. Umgekehrt hieß es von der Staatsanwaltschaft, dass Brunner „alles richtig gemacht habe“. Mit der öffentlichen Ächtung der Angeklagten korrespondierte so eine Hofierung des Opfers zum „Helden“ - unterstützt von den Informationen der Strafverfolgungsbehörden während des Ermittlungsverfahrens. Die in der öffentlichen Diskussion eh anzutreffende Dichotomie in Gut und Böse wurde so angeheizt.

\section{Die Publizierung des Ermittlungsverfahrens}

Eine derartige Publizierung des Ermittlungsverfahrens kannten wir bislang nur bei so genannten Prominenten (siehe Zumwinkel, Kachelmann, „Popsängerin“). Offensichtlich reichen bei spektakulären Straftaten nicht mehr Hauptverhandlung, Verurteilung und Bestrafung aus, um den von den Medien und Politikern geschürten Erwartungen an strafjustizielle Reaktionen $\mathrm{zu}$ entsprechen. Es muss sofort „Kante gezeigt werden“. Die U-Haft gem. $\$ 112$ Abs. 3 GG bei schwerwiegenden Straftaten, insbesondere Tötungsdelikten ohne einen nachgewiesenen gesetzlichen Haftgrund war schon immer ein Einfallstor für derartige dem Strafurteil vorgreifende strafprozessuale Reaktionen in Richtung Öffentlichkeit. Das Bundesverfassungsgericht musste den in nationalsozialistischer Zeit formulierten Haftgrund der „kochenden Volksseele“ verfassungsrechtlich eingrenzen (BVerfGE 19, 350). Angefangen mit einer Mobilisierung der Öffentlichkeit im Strafprozess haben allerdings die Verteidiger. So hat 1984 Rechtsanwalt Kurt Grönewold im so genannten Sternprozess gegen den Angeklagten Kujau die Öffentlichkeit bewusst eingeschaltet, um die Mitverantwortung des Stern für die Veröffentlichung der angeblichen Hitler-Tagebücher deutlich zu machen (Süddeutsche Zeitung vom 14.8.2009). 
Der Journalist Joachim Wagner hat in seiner Publikation „Strafprozessführung über Medien “ die Praktiken von Strafverteidigern und Medien beschrieben und analysiert. Seine Einschätzung aus dem Jahr 1987 erscheint heute wenigstens zum Teil überholt:

„Im Kampf um die öffentliche und veröffentlichte Meinung sind die Staatsanwälte hoffnungslos im Hintertreffen. Das haben viele erkannt. Sie sehen sich aber durch Rolle und Gesetzesauftrag daran gehindert, in der öffentlichen Auseinandersetzung mit Anwälten aktiv um Chancengleichheit zu kämpfen. Sie agieren ängstlich, ungeübt und glauben nach eigenem Bekunden immer noch daran, dass das Gericht dem Druck der Presse widersteht und seine Unabhängigkeit erfolgreich verteidigt. Die Medienöffentlichkeit in Strafverfahren beherrschen daher nach anfänglicher Polizeidominanz eindeutig die Rechtsanwälte." (S. 101) So beschreibt die Spiegel-Gerichtsreporterin Gisela Friedrichsen die heutige Situation wie folgt: „Unstreitig betreiben heute Staatsanwaltschaften im Gegensatz zu früher eine offensivere, bisweilen sogar aggressive Pressearbeit, wenn es sich um Prominente handelt. Gerade bei den Boulevardmedien kommt dies gut an. Und deshalb müssen Strafverteidiger reagieren. “ (ZRP 2010, 263) Und sie tun es, indem in spektakulären Prozessen Strafverteidigern ein Medienanwalt zur Seite gestellt wird.

Die meisten Angeklagten scheuen allerdings die Öffentlichkeit. Nur wenige wissen für ihre Strafverteidigung „Kapital“ über Medienveröffentlichungen zu schlagen.

\section{Die Vorwegverurteilung in der medialen Öffentlichkeit}

Die Vertreter einer offensiven Medienarbeit durch die Strafverfolgungsbehörden können sich somit auf eine Art Verteidigungsstrategie gegenüber den Praktiken der Strafverteidigung berufen. Vor allem gewährt Art. 5 Abs. 1 S. 1 GG einen Informationsanspruch des Bürgers über Medien, konkretisiert in den Landespressegesetzen ( $\sqrt{4})$. Die Öffentlichkeit hat einen Rechtsanspruch, über schwerwiegende Straftaten umgehend informiert zu werden, aber noch nicht über „Straftäter“. Diese werden erst von den Gerichten festgestellt. Bis dahin gilt die Unschuldsvermutung des Art. 6 Abs. 2 MRK, die auch im Rechtsstaatsprinzip des Art. 20 Abs. 3 GG verankert ist. Tatverdächtige als Zeitzeugen („Prominente“) sowie Tatverdächtige in Kriminalfällen, die das Rechtsbewusstsein der Bevölkerung erschüttern, die durch ihre Brutalität nicht nur
Abscheu sondern auch Empörung hervorrufen, müssen sich gefallen lassen, als solche öffentlich identifiziert zu werden (Ostendorf Goltdammer`s Archiv 1980, 457). Ihr Persönlichkeitsschutz muss dann zurücktreten, wobei sie als Verursacher dieses Informationsinteresses in Anspruch genommen werden. Aber der Umfang der Informationen bleibt begrenzt, es bleibt der Grundsatz, dass das Ermittlungsverfahren zum Schutz der Beschuldigten, aber auch um die Beweisführung nicht zu erschweren oder gar unmöglich zu machen, nicht öffentlich und erst die Hauptverhandlung - von Verfahren gegen Jugendliche abgesehen $(\mathbb{S} 48$ Abs. 1 JGG) - öffentlich geführt wird. Wenn die Beweisführung in den Medien vorweggenommen wird, ist die Beweisaufnahme faktisch nicht mehr unmittelbar, auch wenn die Zeugen in der Hauptverhandlung nochmals aussagen. Wenn die Bestrafung in den Medien vorweggenommen wird, muss sich für die Angeklagten der Eindruck der Vorwegverurteilung einstellen. Die unentbehrliche und verfassungsrechtlich garantierte Kontrollfunktion der Medien darf nicht in eine Okkupation von Justizgewalt umschlagen. In anderen Staaten wird versucht, mit einer Bestrafung der Medien wegen contempt of court dem Einhalt zu gebieten. Auch bei uns wurde 1962 mit dem Entwurf zur Reform des StGB ( $\int 452$ : „Störung der Strafrechtspflege“) versucht, repressiv dieses Problem zu lösen. Heute wird ein Verfahrenshindernis (Wohlers StV 2005, 186, 189) oder zumindest ein Strafmilderungsgrund (Hassemer NJW 1985, 1921, 1929) diskutiert. Der Einwand, bei Annahme eines Verfahrenshindernisses hätten es die Medien in der Hand, einen Strafprozess zu sabotieren (Roxin NStZ 1991, 153, 154; Roxin/Schünemann, Strafprozessrecht, 26. Aufl., $\mathbb{} 18$ C Rn. 25), greift dann nicht, wenn die Strafverfolgungsorgane Fehlinformationen streuen oder wichtige Informationen zurückhalten, die zu einer absoluten Vorverurteilung führen. Soweit kann der Vorwurf im „Fall Brunner" nicht erhoben werden, eine gezielte Fehlinformation wurde nicht gegeben. Aber die öffentliche Meinung wurde schon einseitig beeinflusst, wenn nicht gesteuert. Dazu bestand keinerlei Veranlassung, Abscheu und Empörung waren eh bereits durch die Tat und ihre dramatische Folge hervorgerufen. Sie wurden aber gesteigert, keinerlei Entlastung für die Tatverdächtigen sollte aufkommen. Dem Retter der Jugendlichen wurde das Böse der Täter gegenübergestellt. Das erschwert in rechtswidriger Weise die Verteidigungsposition. Ein Be- rufsrichter kann sich von der öffentlichen Vorverurteilung frei machen, aber es ist schwer; ob es auch die Schöffen vermögen, ist fraglich. Zumindest strafschärfende Auswirkungen auf die Straffestsetzung müssen befürchtet werden. Vor allem bleibt in der Öffentlichkeit der Eindruck eines unfairen Verfahrens, wenn erst in der Hauptverhandlung möglicherweise entlastende Momente zutage gefördert werden. Die Staatsanwaltschaft wird dem gesetzlichen Auftrag, auch entlastende Umstände zu ermitteln, nach außen nicht gerecht, mögen diese auch in den Akten stehen: „Die Staatsanwaltschaft hat nicht nur die zur Belastung sondern auch die zur Entlastung dienenden Umstände zu ermitteln und für die Erhebung der Beweise Sorge zu tragen, deren Verlust zu besorgen ist“ ( $\mathbb{S} 160$ Abs. 2 StPO). Einige nehmen vermessen die Bezeichnung „objektivste Behörde der Welt“ für sich in Anspruch. Letztlich schaden die Strafverfolgungsbehörden der Rechtsordnung selbst, wenn sie wichtige Tatumstände bei Offenlegung ansonsten verschweigen. Das Gerechtigkeitsempfinden kann Schaden nehmen. Die Akzeptanz des Urteils sowohl für die unmittelbar Betroffenen, für die Angeklagten und Nebenkläger als auch für die anteilnehmende Öffentlichkeit als gerechte Strafe wird infrage gestellt. Eine solche Informationspolitik schadet dem Rechtsbewusstsein sowie letztlich dem Resozialisierungsanliegen der Verurteilten. Das Bundesverfassungsgericht hat sogar von einem grundrechtlich gewährten Resozialisierungsanspruch gesprochen (BVerfGE 35, 202, 234; 98, 169, 200). Ermittlungsverfahren müssen grundsätzlich nicht öffentlich geführt werden; wenn öffentlich, dann müssen die Informationen zurückhaltend erfolgen; wenn vorweg informiert wird, dann muss korrekt informiert werden. Dies gilt vordringlich für die Informationen durch die Strafverfolgungsbehörden, nachdringlich für die Medienvertreter, die ihrer medialen Verantwortung (Landespressegesetze) gerecht werden müssen. Auch brutale Gewalttäter bleiben Grundrechtsträger, ihnen dürfen der Anspruch der Unschuldsvermutung sowie auf ein faires Verfahren nicht entzogen und die Chance zur Resozialisierung nicht verbaut werden.

\section{Konsequenzen für die Zukunft}

Die mediale Begleitung und Aufarbeitung von Kriminalfällen ist in einer freiheitlichen Gesellschaft unverzichtbar, Bürger haben insoweit einen Anspruch auf Informationen, aber nur auf wahre Informationen. Fehlin- 
formationen sind nicht nur nicht rechtlich gedeckt, sie verstoßen auch gegen die Wahrheitsverpflichtung aller, die Informationen herausgeben und verbreiten. Hierbei kommt es nicht auf den Wortlaut an, sondern auf den Eindruck, den die Mitteilung hervorruft bzw. geeignet ist hervorzurufen (BGHZ 27, 338). Unschuldsvermutung, das Recht auf faires Verfahren sowie der Resozialisierungsanspruch, die schon für eine wahre Information zur Zurückhaltung zwingen, stehen unwahren Informationen erst recht entgegen.

Strafrechtliche Konsequenzen sind nicht angezeigt. Jede strafrechtliche Inpflichtnahme von Medien stößt sich mit dem grundrechtlich verbürgten Recht der Medienfreiheit (Art. 5 Abs. 1 S. 2 GG). Der Ausfluss des Informationsanspruchs der Öffentlichkeit (Art. 5 Abs. 1 S. 1 GG) sowie praktische Einwände gegen die Einrichtung eines strafrechtlichen Nebenkriegsschauplatzes stehen auch einer strafrechtlichen Kontrolle der Strafverfolgungsorgane entgegen. Schon die Tatbestände der Rechtsbeugung ( $\$ 339$ StGB) sowie der Verfolgung Unschuldiger $(\mathbb{S} 344 \mathrm{StGB})$ haben sich in der Praxis als stumpfe Schwerter erwiesen.

Es bleiben strafprozessuale Konsequenzen - von der Eigeninitiative Betroffener abgesehen, im Wege eines Rechtsschutzes über die \$\$ 23 ff. EGGVG oder $\$ 40$ VwGO sowie der Geltendmachung eines Schadensersatzanspruchs im Wege der Amtshaftung sowie eines eventuell vorbeugenden Unterlassungsanspruchs ihr Recht zu suchen.

Soweit Richter an der Herausgabe (teil) unwahrer Informationen beteiligt sind, drängt sich die Besorgnis der Befangenheit auf ( $\$ 24$ Abs. 2 StPO). Dies gilt nach Einreichung der Anklageschrift auch für das Unterlassen von Richtigstellungen, soweit von Polizei und/oder Staatsanwaltschaft danach Fehlinformationen herausgegeben werden. Ab Einreichung der Anklageschrift ist die Strafjustiz verantwortlich für das Strafverfahren. Die Ausweitung der Ablehnung wegen Besorgnis der Befangenheit auf die Staatsanwaltschaft wird von der h. M. abgelehnt, die Auswechslung eines für befangen erklärten Staatsanwalts begründet in der Praxis keinen Schutz vor derartigen Fehlinformationen.

Effektiver erscheinen die Überlegungen, wie bei sonstigen schweren Verfahrensverstößen gegen das fair-trial-Prinzip, z. B. wegen einer von den Strafverfolgungsbehörden bzw. der Strafjustiz zu verantwortenden Überlänge des Verfahrens sowie bei „Verführung“ von unbescholtenen Bürgern zu Straftaten durch verdeckte Ermittler der Polizei und/oder VPersonen, hier ein Verfahrenshindernis, zumindest eine obligatorische Strafmilderung im Wege der Anrechnung auf die Strafverbüßung zu begründen. $\mathrm{Ob}$ sich diese Optionen realisieren, muss abgewartet werden. Bis dahin bleibt der Appell an das Berufsethos der Betroffenen entsprechend der Nr.
23 Richtlinien für das Straf- und Bußgeldverfahren. Hiernach darf die Unterrichtung der Öffentlichkeit über Medien „weder den Untersuchungszweck gefährden noch dem Ergebnis der Hauptverhandlung vorgreifen; der Anspruch des Beschuldigten auf ein faires Verfahren darf nicht beeinträchtigt werden. “ Diese rechtlich-ethische Verpflichtung zu einer wahren Informationspolitik ist in der Praxis leichter einlösbar, wenn die Herausgabe von Presseinformationen nicht den unmittelbar beteiligten Strafverfolgern überlassen wird, sondern - wie eigentlich auch üblich - einem verantwortlichen Medienvertreter in der Staatsanwaltschaft sowie bei Gericht. Damit einher ist das Verbot aufzustellen, in Ermittlungsverfahren sowie in der Hauptverhandlung über allgemein verbreitete Medieninformationen hinaus Einzelauskünfte an Medienvertreter zu geben. Allgemeine Presseinformationen sollten sich auf die Zurückweisung unwahrer Behauptungen bzw. Verdächtigungen von der Angeklagtenseite oder der Nebenklage sowie von Medienvertretern begrenzen. Anklage und Hauptverhandlung müssen für sich selbst sprechen. Die Wahrheitssuche im Strafprozess findet so ihre Ergänzung in der Verpflichtung zu einer wahrheitsgemäßen Information über den Strafprozess.

\section{Fußnote}

1 Für die Aufarbeitung der rechtlichen Diskussion bedanke ich mich bei meinem Mitarbeiter Felix Doege.

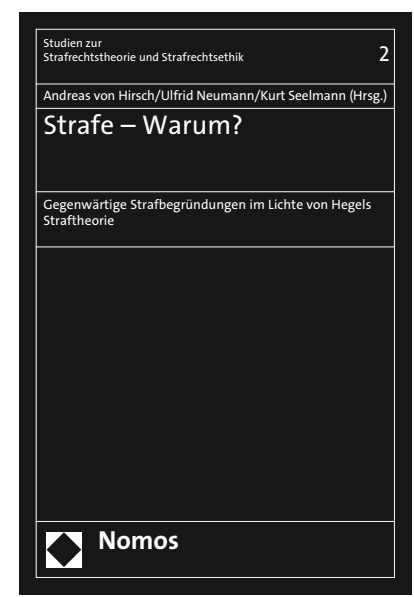

\section{Strafe - Warum?}

Gegenwärtige Strafbegründungen im Lichte von Hegels Straftheorie

Herausgegeben von Prof. Dr. Dr. h.c. Andreas von Hirsch, Prof. Dr. Dr. h.c. Ulfrid Neumann und Prof. Dr. Dr. h.c. Kurt Seelmann

2011, 234 S., brosch., 59,- €, ISBN 978-3-8329-6918-9

(Studien zur Strafrechtstheorie und Strafrechtsethik, Bd. 2)

Erscheint ca. September 2011

nomos-shop.de/14016

Warum muss Strafe sein? Aktuellen Strafbegründungstheorien, insbesondere solchen, die in der Strafe einen Tadel sehen, wird Hegels Straftheorie vom Tadel als Wiederherstellung eines Rechtsverhältnisses gegenübergestellt. Schließlich gilt es zu klären, ob Hegels Sicht der Dinge die zeitgenössischen Ansätze zum Verständnis der Strafbegründung befruchten kann. 